\title{
Fatal Systemic Mucormycosis after Rabbit Anti-Thymocyte Globulin Therapy in a Severe Aplastic Anemia Patient
}

\author{
${ }^{1}$ Department of Hematology/Oncology, Teikyo University School of Medicine, Japan \\ ${ }^{2}$ Department of Pathology, Teikyo University School of Medicine, Japan \\ ${ }^{3}$ Department of Infection Control and Prevention, Teikyo University Hospital, Japan
}

Haruko Tashiro MD, PhD ${ }^{1^{*}}$, YasutoshiOshima ${ }^{2}$, Ritsu Sumiyoshi ${ }^{1}$, Takuji Matsuo ${ }^{1}$, Tadashi Yamamoto ${ }^{1}$, Kensuke Matsumoto ${ }^{1}$, Jun Ooi ${ }^{1}$, Naohisa Matsunaga ${ }^{3}$, Yoshinao Kikuchi ${ }^{2}$ and Naoki Shirafuji ${ }^{1}$

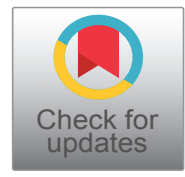

*Corresponding author: Haruko Tashiro, MD, PhD, Department of Hematology/Oncology, Teikyo University School of Medicine, 2-11-1 Kaga, Itabashi-ku, Tokyo 173-8606, Japan, Tel: +81-3-3964-1211, Fax: +81-3-3964-1600

\begin{abstract}
Survival rates for patients with severe aplastic anemia have greatly improved with the development of better supportive care including transfusion strategies and the availability of anti-fungal agents. However, invasive fungal infection remains the main cause of death and increases mortality in severe aplastic anemia patients. Among invasive fungal infections, mucormycosis is one of the fatal diseases in immunocompromised patients. We report the case of a 72-year-old man with severe aplastic anemia treated with immunosuppressive therapy consisting of rabbit anti-thymocyte globulin, cyclosporine, and steroids. The patient developed pneumonia, which did not respond to broad-spectrum antibiotics or echinocandin anti-fungal agents. The pneumonia rapidly progressed and the patient died of multi-organ failure on day 38 after the start of immunosuppressive therapy. The autopsy findings revealed that multiple organs, i.e., lungs, kidneys, heart, liver, spleen, thyroid, and pancreas, were invaded by fungi, and massive coagulative necrosis and thrombotic vessels were found in those organs. Our cultures of a tissue specimen from the patient's left lung were positive for Rhizomucor. We discuss the current problems related to severe aplastic anemia treatment in Japan.
\end{abstract}

\section{Keywords}

Aplastic anemia, Mucormycosis, ATG

\section{Introduction}

Due to advancements in supportive cares including better transfusion strategies and the availability of antifungal agents, survival rates for patients with severe aplastic anemia (SAA) have greatly improved [1]. However, infections, especially invasive fungal infections (IFI), remain the main cause of death in this patient population [2,3]. In contrast to neutropenia in cancer patients, which is often reversible and of short duration, neutropenia in SAA patients is longer and sometimes irreversible. It is known that prolonged neutropenia is the dominant risk factor in the development of IFI $[1,2,4]$. In addition to the neutropenia caused by SAA itself, immunosuppressive treatments for SAA further increase the risk of infection. For SAA patients, an Aspergillus active azole, preferably itraconazole or posaconazole, is currently recommended for use as prophylaxis by the British Society for Standards in Haematology [5]. Posaconazole is one of the few drugs also active against Mucorales. Mucorales were reported to cause pulmonary, rhinocerebral, cutaneous, gastrointestinal, and disseminated infections [1]. The mortality rate is high in immunocompromised patients with disseminated mucormycosis [1]. However, in Japan, posaconazole is not clinically available, and due to the erratic bioavailability of the oral solution the use of this drug has limitations [6].

Here, we report a patient who developed fatal systemic mucormycosis after rabbit anti-thymocyte globulin ( $r A T G$ ) treatment and discuss the current problems related to SAA treatment in Japan.

Citation: Tashiro H, Oshima Y, Sumiyoshi R, Matsuo T, Yamamoto T, et al. (2019) Fatal Systemic Mucormycosis after Rabbit Anti-Thymocyte Globulin Therapy in a Severe Aplastic Anemia Patient. Int J Blood Res Disord 6:039. doi.org/10.23937/2469-5696/1410039

Accepted: May 14, 2019: Published: May 16, 2019

Copyright: (c) 2019 Tashiro H, et al. This is an open-access article distributed under the terms of the Creative Commons Attribution License, which permits unrestricted use, distribution, and reproduction in any medium, provided the original author and source are credited. 


\section{Case Report}

A 72-year-old Japanese male was referred to our hospital due to pancytopenia. White blood cell (WBC) and neutrophil counts in peripheral blood were $2.1 \times$ $10^{9} / \mathrm{L}$ and $0.4 \times 10^{9} / \mathrm{L}$, respectively. He also had anemia $(\mathrm{Hb} 6.4 \mathrm{~g} / \mathrm{dL}$ ) and thrombocytopenia (Platelet 7 $\times 10^{9} / \mathrm{L}$ ). The absolute reticulocyte count was $1.79 \times$ $10^{9} / \mathrm{L}$. The patient received transfusions of red blood cells and platelet concentrate regularly once or twice a week. Bone marrow study revealed severe hypoplastic marrow with increased fat content, and chromosomal analysis showed a normal karyotype. As the patient was ineligible for magnetic resonance imaging due to a lumbar fusion surgical history, the bone marrow was evaluated with Indium $\left({ }^{111} \mathrm{I} \mathrm{n}\right)$ Chloride bone marrow scintigraphy. The bone marrow scintigraphy found minimal bone marrow activity only in vertebrae, with no activity observed in other bony tissues. From these results, we diagnosed SAA. During the diagnostic workup, bacterial pneumonia developed in the right upper lobe. Although we were not able to define the causal pathogen, the pneumonia responded to cefepime. After resolving the pneumonia, the patient received immunosuppressive therapy (IST) consisting of rATG $(3.75 \mathrm{mg} /$ $\mathrm{kg}$ for 5 days), cyclosporine, and steroids as treatment for SAA. $50 \mathrm{mg} /$ day of micafungin was administered for prophylaxis against fungal infections. Although there were no severe adverse events during administration of rATG, hematological recovery was delayed. The neutrophil count was below $0.2 \times 10^{9} / \mathrm{L}$ for more than 2 weeks despite the administration of granulocyte-colony stimulating factor. On day 20 after starting IST, Enterococcus faecium bacteremia with low-grade fever developed and vancomycin was added to cefepime. At this point, WBC and neutrophil counts were $0.20 \times 10^{9} / \mathrm{L}$ and 0.18 $\times 10^{9} / \mathrm{L}$, respectively. Although follow-up blood cultures found no positive growth for bacteria or fungi, the fever worsened to a high-grade fever. We switched antibiotics from cefepime to doripenem and escalated the dose of micafungin from $50 \mathrm{mg}$ to $150 \mathrm{mg} /$ day. A chest computed tomography (CT) scan on day 30 after IST found an old inflammatory change in the upper lobe of the right lung and a fresh consolidation in the upper lobe of the left lung without typical fungal infection signs, such as halo sign, air-crescent sign, or cavitary lesions within an area of consolidation (Figure 1A). Multiple sputum cultures found no meaningful results. The patient required mechanical ventilation support in an intensive care unit beginning on day 33 of IST. Although there was no serological evidence of fungal infection (i.e., elevation of Aspergillus antigen, Candida antigen, or $\beta$-D-glucan) and the clinical findings did not fulfil even possible IFI according to the modified European Organization for the Re-
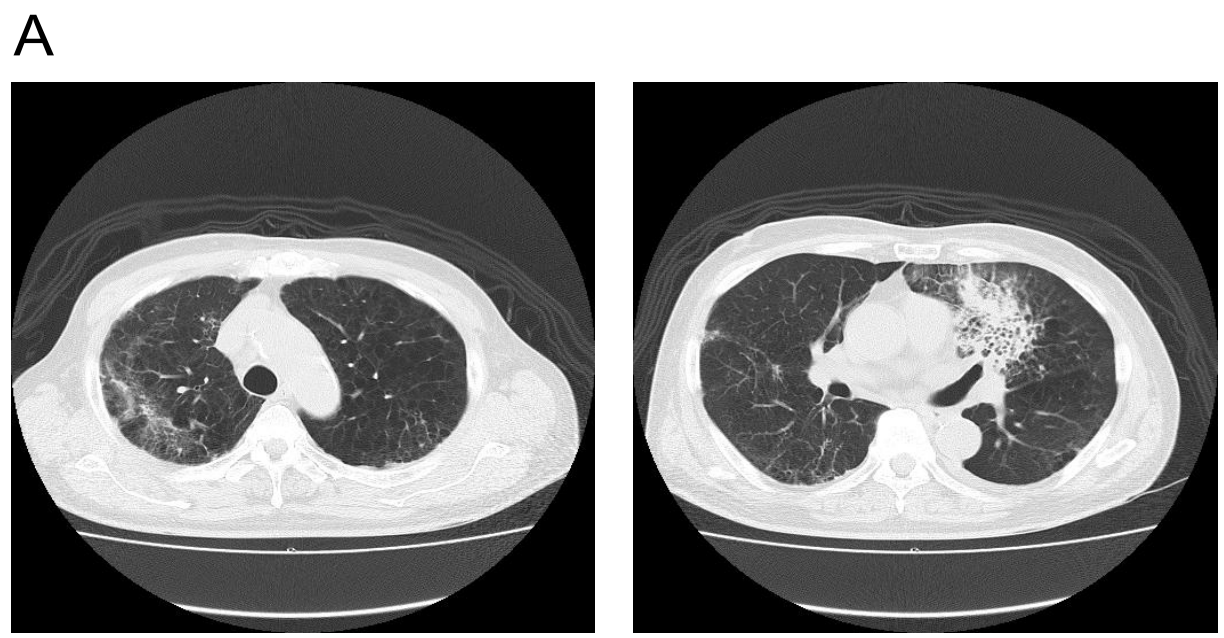

B

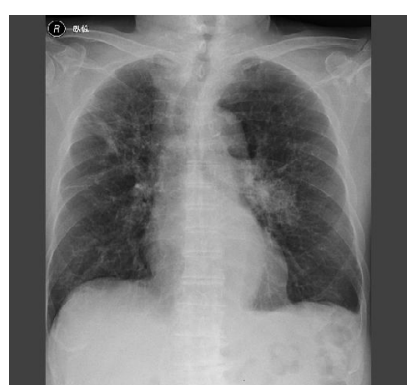

Day 30

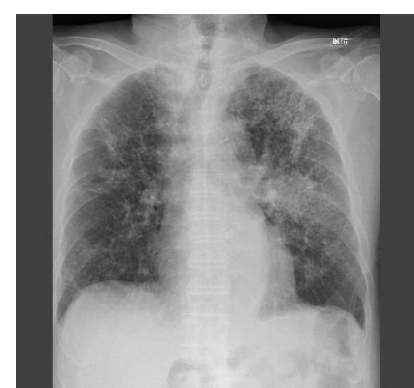

Day 33

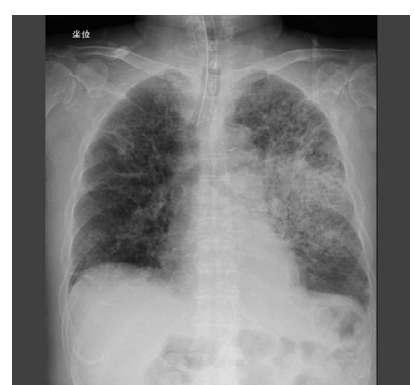

Day 36

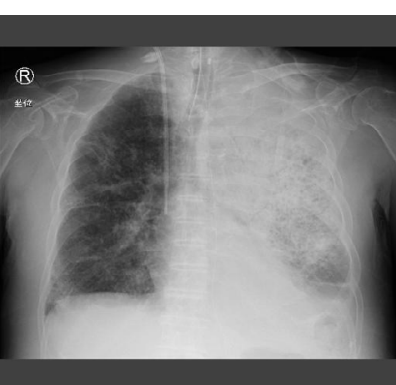

Day 38

Figure 1: A) Chest CT scan on day 30 showed an old inflammatory change in the right upper lobe and a fresh consolidation in the left upper lobe without typical fungal infection signs; B) Chest $X$ rays on days 30, 33, 36, and 38 after starting immunosuppressive therapy. The consolidation on the left lung expanded rapidly. 


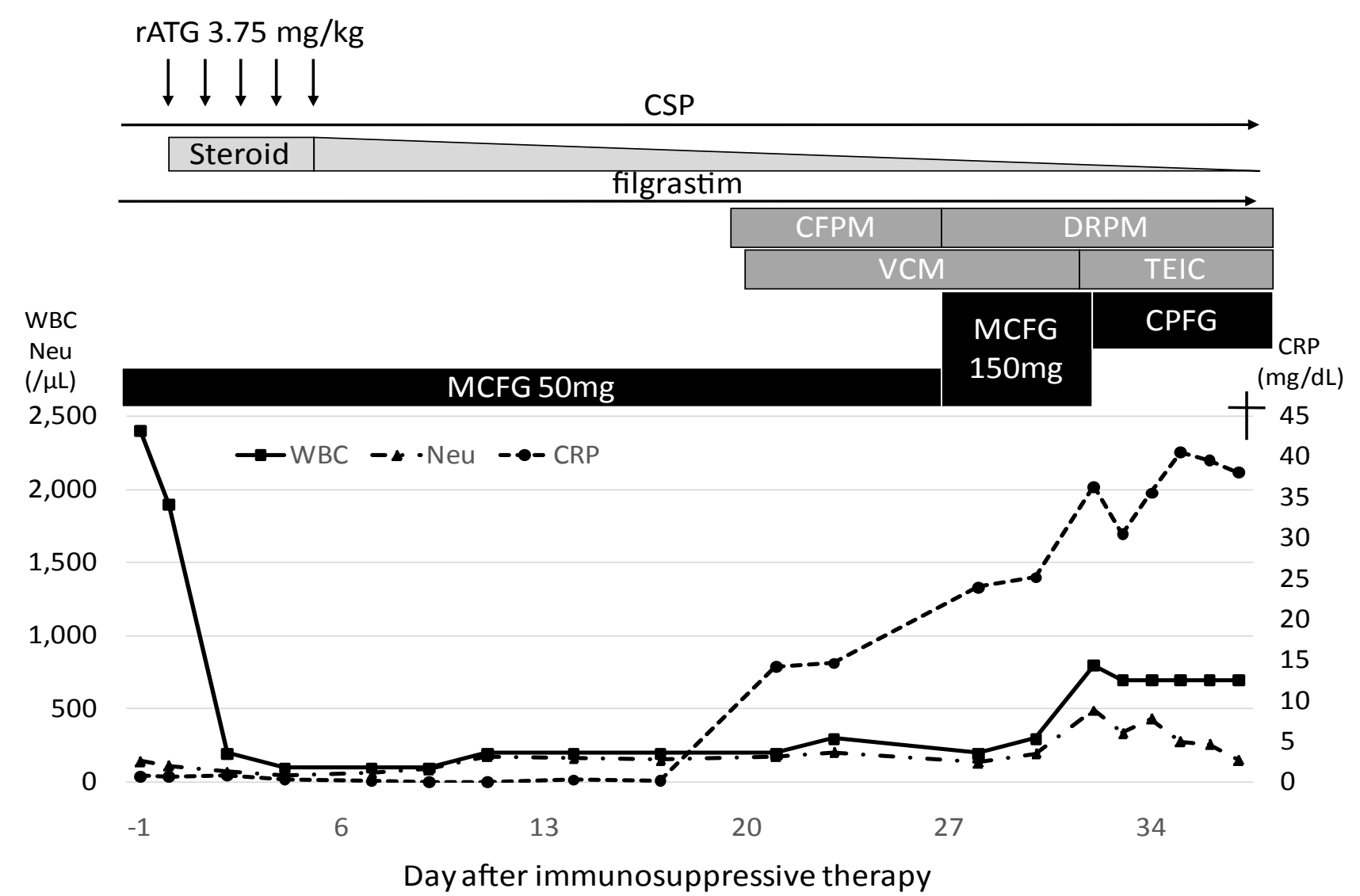

Figure 2: Clinical course of the patient.

rATG: Rabbit Anti-Thymocyte Globulin; CSP: Cyclosporine A; CFPM: Cefepime; DRPM: doripenem; VCM: Vancomycin; TEIC: Teicoplanin; MCFG: Micafungin; CPFG: Caspofungin; WBC: White Blood Cell Count; Neu: Absolute Neutrophil Count; CRP: C-Reactive Protein.

search and Treatment of Cancer/Mycoses Study Group criteria [7], the pneumonia continued to worsen despite administration of broad-spectrum antibiotics. Improvement was not observed after we replaced micafungin with caspofungin on day 31 . The patient's pneumonia progressed rapidly (Figure $1 \mathrm{~B}$ ) and severe disseminated coagulopathy developed on day 36. Death occurred due to multi-organ failure on day 38 . On the day the patient died, zygomycetes were detected in a sputum culture. The clinical course is summarized in Figure 2.

An autopsy was performed, and multiple organs, i.e., lungs (Figure $3 \mathrm{~A}$ and Figure 3B), kidneys, heart (Figure $3 C$ and Figure 3D), liver, spleen, thyroid, and pancreas (Figure $3 \mathrm{E}$ and Figure $3 \mathrm{~F}$ ) showed massive coagulative necrosis and thrombotic vessels containing fungus. We cultured a piece of tissue from the patient's left lung, which revealed the growth of Rhizomucor. The bone marrow remained severely hypocellular with increased fat content in the marrow space.

\section{Discussion}

Despite the similar aggressiveness and mortality, the prophylaxis for mucormycosis is not well established compared to Aspergillus infection. Although mucormycosis is an uncommon infection, it was recently recognized as an emerging pathogen that causes IFI [8]. Mucormycosis is a high mortality disease and requires a high index of suspicion in SAA patients [1].

It is well known that prolonged neutropenia is often observed in SAA patients and is a risk factor of IFI. In addition, one risk factor for developing mucormycosis is an excess of iron. Iron is an essential element for cell growth and development, and its acquisition is a critical step in the causative organisms' pathological mechanism. While deferoxamine is an iron chelator drug used to prevent excess of iron in patients frequently receiving red blood transfusions, this drug also predisposes patients to mucormycosis by acting as a siderophore, which supplies previously unavailable iron to the fungus [9]. Although our patient was not treated with deferoxamine, his serum iron and ferritin levels were high (231 $\mu \mathrm{g} / \mathrm{dL}$ and $2635 \mathrm{ng} / \mathrm{mL}$, respectively) and these should be considered as being high risk for mucormycosis. The patient received $50 \mathrm{mg} /$ day of micafungin as prophylaxis for fungal infections. After the pneumonia developed, which was refractory to broad-spectrum antibiotics, we planned to use Liposomal Amphotericin B (L-AMB) to cover Aspergillus and Mucorales. L-AMB is considered to have less nephrotoxicity than other forms of amphotericin B $[10,11]$. However, combined with other drugs, such as foscarnet and/or cyclosporine, a higher incidence of renal injury by L-AMB was reported [12]. Since our patient had renal dysfunction, was receiving treatment with cyclosporine, and did not have a high sus- 

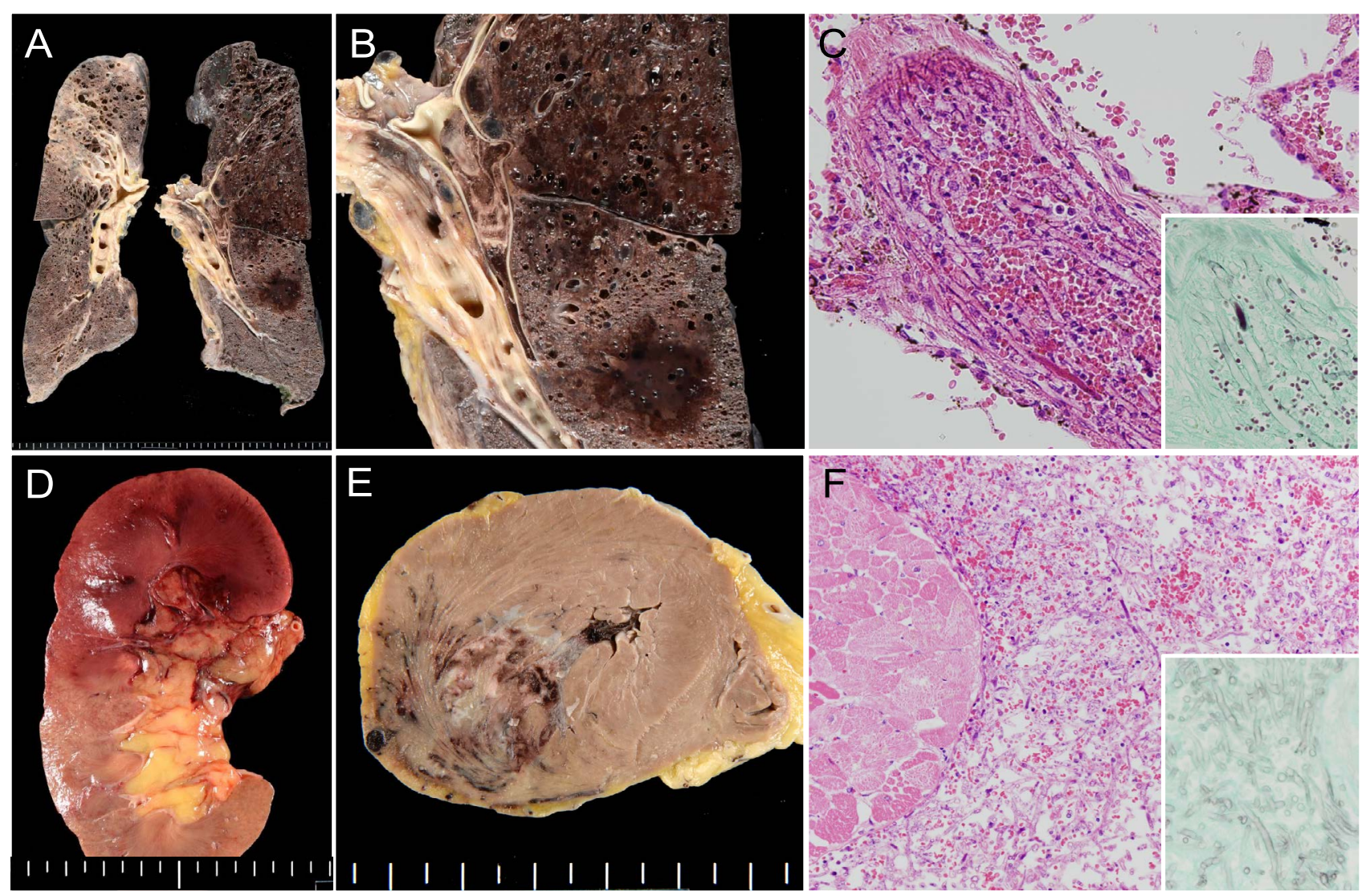

Figure 3: Autopsy finding demonstrating systemic mucormycosis.

A) Gross finding of representative slices of the bilateral lungs; B) Hemorrhagic infarction and pulmonary artery thrombosis of the left lung; C) Histologic finding of pulmonary artery embolization caused by mucor hyphae (inset: Grocott's staining showing non-septate fungal hyphae); D) Gross finding of renal infarction caused by fungal embolization; E) Myocardial hemorrhagic infarction; F) Mucor hyphae permeating between necrotic myocardium (inset: Grocott's staining).

picion of mucormycosis, we decided not to use L-AMB and instead treated him with caspofungin. Caspofungin is active against Aspergillus, but not Mucorales. Our case suggests that anti-mucor therapy is required for patients with prolonged neutropenia who have developed broad-spectrum antibiotics-resistant infection, even in cases of renal dysfunction. Isavuconazole, a new broad-spectrum triazole anti-fungal agent, shows anti-mucor activity [13]. It was reported that this drug is active against Mucorales with efficacy similar to amphotericin B and is well tolerated [14,15]. Since mucormycosis progresses rapidly, prophylaxis may be more important than empirical therapy for SAA patients. Since SAA patients are at high risk of mucormycosis for the above reasons, anti-mucor prophylaxis should ideally be initiated early in the hospital course.

Another problem with SAA treatment is that currentIy in Japan only rabbit ATG is clinically available, but not horse ATG (hATG). Several clinical studies showed the superiority of hATG over rATG $[16,17]$. Scheinberg, et al. reported that in a randomized prospective study, rATG plus cyclosporine was inferior to hATG plus cyclosporine when administered as a first line treatment for SAA. The hematological response rate at 6 months was $68 \%$ with hATG and $37 \%$ with rATG [16]. Since prolonged hematological recovery is critical for patients, the clinical avail- ability of hATG in Japan is required.

In conclusion, we experienced an SAA patient who was treated with rATG and developed disseminated mucormycosis uncovered by autopsy. We should always have a high suspicion of mucormycosis for antibiotic-resistant infection in aplastic anemia patients, and should not delay the initiation of anti-mucor therapy in suspicious cases. Prophylactic therapy against Mucorales might be required for high-risk patients.

\section{Conflict of Interest}

The authors declare no conflicts of interest.

\section{Consent}

Written informed consent was obtained from the patient's family to publish the case report.

\section{References}

1. Valdez JM, Scheinberg P, Young NS, Walsh TJ (2009) Infections in patients with aplastic anemia. Semin Hematol 46: 269-276.

2. Weinberger M, Elattar I, Marshall D, Steinberg SM, Redner RL, et al. (1992) Patterns of infection in patients with aplastic anemia and the emergence of Aspergillus as a major cause of death. Medicine (Baltimore) 71: 24-43.

3. Valdez JM, Scheinberg P, Nunez O, Wu CO, Young NS, 
et al. (2011) Decreased infection-related mortality and improved survival in severe aplastic anemia in the past two decades. Clin Infect Dis 52: 726-735.

4. Torres HA, Bodey GP, Rolston KV, Kantarjian HM, Raad II, et al. (2003) Infections in patients with aplastic anemia: Experience at a tertiary care cancer center. Cancer 98: 8693.

5. Killick SB, Bown N, Cavenagh J, Dokal I, Foukaneli T, et al. (2016) Guidelines for the diagnosis and management of adult aplastic anaemia. Br J Haematol 172: 187-207.

6. Cornely OA, Vehreschild JJ, Ruping MJ (2009) Current experience in treating invasive zygomycosis with posaconazole. Clin Microbiol Infect 5: 77-81.

7. Ascioglu S, Rex JH, de Pauw B, Bennett JE, Bille J, et al. (2002) Defining opportunistic invasive fungal infections in immunocompromised patients with cancer and hematopoietic stem cell transplants: An international consensus. Clin Infect Dis 34: 7-14.

8. Roden MM, Zaoutis TE, Buchanan WL, Knudsen TA, Sarkisova TA, et al. (2005) Epidemiology and outcome of zygomycosis: A review of 929 reported cases. Clin Infect Dis 41: 634-653.

9. Boelaert JR, de Locht M, Van Cutsem J, Kerrels V, Cantinieaux $B$, et al. (1993) Mucormycosis during deferoxamine therapy is a siderophore-mediated infection. In vitro and in vivo animal studies. J Clin Invest 91: 1979-1986.

10. Falci DR, da Rosa FB, Pasqualotto AC (2015) Comparison of nephrotoxicity associated to different lipid formulations of amphotericin B: A real-life study Mycoses 58: 104-112.

11. Wade RL, Chaudhari P, Natoli JL, Taylor RJ, Nathanson $\mathrm{BH}$, et al. (2013) Nephrotoxicity and other adverse events among inpatients receiving liposomal amphotericin $B$ or amphotericin B lipid complex. Diagn Microbiol Infect Dis 76: 361-367.

12. Stanzani M, Vianelli N, Cavo M, Maritati A, Morotti M, et al. (2017) Retrospective cohort analysis of liposomal amphotericin B nephrotoxicity in patients with hematological malignancies. Antimicrob Agents Chemother 61.

13. Miceli MH, Kauffman CA (2015) Isavuconazole: A New Broad-Spectrum Triazole Antifungal Agent. Clin Infect Dis 61: 1558-1565.

14. Marty FM, Ostrosky-Zeichner L, Cornely OA, Mullane KM, Perfect JR, et al. (2016) Isavuconazole treatment for mucormycosis: A single-arm open-label trial and casecontrol analysis. Lancet Infect Dis 16: 828-837.

15. Graves B, Morrissey CO, Wei A, Coutsouvelis J, Ellis $\mathrm{S}$, et al. (2016) Isavuconazole as salvage therapy for mucormycosis. Med Mycol Case Rep 11: 36-39.

16. Scheinberg $P$, Nunez $O$, Weinstein B, Biancotto A, Wu CO, et al. (2011) Horse versus rabbit antithymocyte globulin in acquired aplastic anemia. N Engl J Med 365: 430-438.

17. Hayakawa J, Kanda J, Akahoshi Y, Harada N, Kameda $\mathrm{K}$, et al. (2017) Meta-analysis of treatment with rabbit and horse antithymocyte globulin for aplastic anemia. Int $\mathrm{J}$ Hematol 105: 578-586. 